\title{
RESPONSABILIDADE AMBIENTAL, SINALIZAÇÃO E DESEMPENHO ECONÔMICO: UMA ANÁLISE A PARTIR DO CASSO DAS FRANQUIAS
}

\author{
Helder de Souza Aguiar \\ Doutorando em Administração \\ Universidade de São Paulo - FEA USP \\ São Paulo - São Paulo - Brasil \\ helderaguiar@usp.br \\ Bruno Brandão Fischer \\ Doutor em Economía y Gestión de la Innovación \\ Universidade Estadual de Campinas - Unicamp \\ Limeira - São Paulo - Brasil \\ bruno.fischer@fca.unicamp.br \\ Flavia Consoni \\ Doutora em Política Científica e Tecnológica \\ Universidade Estadual de Campinas - Unicamp \\ Campinas - São Paulo - Brasil \\ flavia@ige.unicamp.br
}

\section{RESUMO}

O mercado de franquias do Brasil tem se consolidado e mostrado fôlego de crescimento invejável nos últimos anos. Os atores franqueados, o franqueador e os clientes finais compõem a tríade que sustenta este ramo de atividades. Não obstante, a dinâmica de relacionamentos entre estes agentes impõe certos desafios para novos empreendedores. Na busca por criar valor mercadológico e diferenciação, o tema de responsabilidade ambiental apresenta relevância crescente no universo das franquias. Com base nesse contexto, este estudo se dedica a avaliar a associação entre sinalização de atributos ambientais por parte das franquias e seus resultados econômicos de curto prazo em termos de faturamento, lucratividade e tempo de retorno dos investimentos. Utilizando-se de uma amostra de franquias operantes no Brasil, estimam-se modelos de regressão para práticas ambientais corporativas próprias e outorgadas por órgãos independentes. Com base nos dados obtidos por meio da estimação de modelos de regressão, não foi possível afirmar que as empresas emissoras de sinais relativos à questão ambiental alcançaram desempenho superior nos critérios avaliados. Com efeito, sinais significativos foram associados somente com tempos maiores de retorno dos investimentos realizados. Ainda que inesperados, estes resultados apresentam alinhamento com parte da literatura dedicada a estes fenômenos. Implicações destas evidências para o caso das franquias são elencadas e discutidas.

Palavras-chave: Franchising; Franquia; Franqueados; Mecanismos de sinalização; Sustentabilidade.

\section{ENVIRONMENTAL RESPONSIBILITY, SIGNALING AND ECONOMIC PERFORMANCE: AN ANALYSIS FROM FRANCHISES CASES}

\begin{abstract}
The franchising market in Brazil has presented a consolidation pattern and strong growth trends in recent years. The franchised actors, the franchisor, and end customers make up the triad that sustains this activity. Nevertheless, the dynamics of relationships between these agents imposes challenges for entrepreneurs. In the search for creating market value and differentiation, the field of environmental responsibility has become increasingly relevant for franchises. Based on this proposal, this article is dedicated to evaluating the association between environmental attributes' signaling strategies by franchises and their short-term economic results regarding revenues, profitability and time of return on investments. Using a sample of franchises operating in Brazil, regression models are estimated for independent environmental practices and certifications granted by third-parties. Based on the data obtained through statistical estimations we found that companies sending signals related to environmental sustainability achieved a superior performance in the evaluated criteria. In fact, significant coefficients were associated only with greater times of return on investments. Although unexpected, these results are in line with part of the literature dedicated to these phenomena. Implications of this evidence for the case of franchises are listed and discussed.
\end{abstract}

Key words: Franchising: Franchise; Franchisors; Signaling mechanisms; Sustainability. 


\section{INTRODUÇÃO}

O mercado de franquias do Brasil tem se consolidado e mostrado fôlego de crescimento invejável apesar das constantes crises econômicas, internas ou externas, neste início de século XXI. Tal movimento se reflete nos dados divulgados pela principal organização da categoria no Brasil, a Associação Brasileira de Franchising [ABF], que contabilizou 3.039 redes de franquias dos mais diversos tamanhos e áreas de atuação em operação no Brasil em 2016 (ABF, 2017). Também vale destacar uma tendência de ampliação e diversificação de redes de franquia: o número de unidades do sistema que em 2003 foi de 56.564, se ampliou para 144.074 em 2016 (ABF, 2017). A média de unidades por rede, que em 2003 foi de 83,4, o que representava 47,4 unidades por rede em 2016, em um claro movimento de ampliação e diversificação das ofertas de redes de franquias no mercado.

No negócio das franquias, os atores franqueados, os clientes finais e o franqueador compõem a tríade que sustenta o sistema. Não obstante, a dinâmica de relacionamentos entre estes agentes impõe certos desafios para novos empreendedores. Partindo-se dos dados elencados acima, reforça-se um maior nível de concorrência agregada entre as franquias, de forma que as redes necessitam se diferenciar umas das outras e mostrar uma maior atratividade para conquistarem novos franqueados. Entre esses diferenciais, é necessário mostrar que as redes são rentáveis, que possuem um modelo de negócio sustentável do ponto de vista econômico, que estão bem posicionadas no mercado e que possuem uma reputação positiva perante o mercado (Brandt, 2007; Monge \& Huerta, 2015; Aguiar, Pauli, Yu, \& Nascimento, 2016).

É no âmbito das diversas abordagens adotadas pelas empresas para criar valor no mercado, tornar sua marca atrativa e se diferenciar da concorrência, que está o tema da responsabilidade ambiental corporativa (Dahlsrud, 2008; Murray, 2004). Esta abordagem se alinha a uma demanda cada vez mais latente da sociedade. Diferentemente da visão neoclássica - a qual pontua que responsabilidades socioambientais são deveres do Estado e da sociedade e não das firmas (Friedman, 1970) - cada vez mais os consumidores têm cobrado das organizações um comportamento mais em linha com tais responsabilidades.

Esta posição é reforçada pela abordagem postulada pelo triple bottom line de que será cada vez mais difícil para as empresas fazerem negócio, tendo em vista "[...]o empobrecimento dos clientes, a degradação do meio ambiente, a falência de sistemas políticos e a dissolução das sociedades" (Elkington, 1997, p. 109). De acordo com esta percepção, surge a necessidade de as organizações considerarem questões de performance ambiental e social, além da financeira. Consequentemente, busca-se conciliar as expectativas das pessoas, do planeta e da necessidade do lucro.

Não obstante, desde um ponto de vista empírico, a questão de associação entre desempenho ambiental corporativo e desempenho financeiro apresenta resultados contraditórios (Albertini, 2013; Endrikat, Guenther \& Hoppe, 2014; Huenther \& Hoppe, 2014; Testa \& D’Amato, 2017) . Para a dinâmica econômica das franquias, este é um ponto de elevada sensibilidade estratégica. De acordo com o argumento exposto neste artigo, mecanismos que impactem na receita potencial do negócio influenciam não somente os resultados diretos da rede, mas também sua própria atratividade para novos empreendedores e, assim, sua competitividade no longo prazo.

Conforme abordado nos trabalhos seminais da economia da informação (Akerlof, 1970; Spence, 1973), existe um elevado nível de assimetria de informação entre os agentes econômicos, particularmente quanto aos atributos intrínsecos dos produtos/serviços transacionados. Neste cenário, o uso de mecanismos de sinalização corresponde às atividades realizadas pelos agentes para informar o mercado sobre características relevantes para sua diferenciação (Dewally \& Ederington, 2006). Dessa forma, o uso de mecanismos de sinalização de sustentabilidade ambiental serve ao propósito de gerar maiores níveis de competitividade nas empresas que fazem uso destes expedientes, partindo-se da hipótese de que as características da demanda valorizam tais atributos.

Contudo, a literatura neste campo orientada ao caso de franquias ainda está em consolidação. Assim, este artigo busca explorar evidências empíricas acerca de estratégias de 
atuação que privilegiem iniciativas voltadas à responsabilidade ambiental e sua associação com resultados financeiros das empresas. O questionamento norteador desta investigação trata de inquirir se franquias ambientalmente sustentáveis envolvidas em esforços de sinalização vivenciam um desempenho econômico superior.

Com vistas a operacionalizar esta proposta, tomamos uma amostra de franquias fornecida pela ABF/Afras e identificamos mecanismos de sinalização de atividades ambientalmente sustentáveis em duas instâncias: sinalização corporativa própria (programas e atividades informadas pelas empresas e vinculadas às suas marcas e ações) e sinalização outorgada por órgãos independentes (selos, certificações e prêmios de sustentabilidade ambiental). Para a elaboração deste estudo, foram analisadas 167 empresas envolvidas com atividades de franquia (anos 20132014).

Esse artigo encontra-se organizado em sete seções, incluindo esta Introdução. A seção 2 explora a noção de identidade da marca que permeia a relação entre o franqueador e o franqueado e deve ser consistente com a proposta de franquia que se busca. Esta proposta se manifesta a partir dos instrumentos de sinalização, os quais enviam para o mercado "mensagens" associadas a atributos que ficam associados aos produtos/serviços que as franquias comercializam, conforme exposto na seção 3. Esta seção também avança ao estabelecer as hipóteses da pesquisa que são testadas a partir de método e variáveis especificados na seção 4. A seção 5 analisa os resultados da aplicação de modelos regressivos multivariados e a seção 6 discute os achados empíricos. A seção 7 encerra com as considerações finais da pesquisa.

\section{ASPECTOS INTRODUTÓRIOS DA GESTÃO ESTRATÉGICA EM FRANQUIAS}

Franchising se caracteriza basicamente pela padronização de um negócio testado em mercado, centrado em produtos ou serviços (Stanworth \& Dandridge, 1994). Uma unidade franqueada faz uso da marca da rede e se apresenta como um prolongamento dela, difundindo esta identidade que deve ser geral para todas as unidades. Usar a marca da rede é um pilar desse sistema. Assim, mostrar-se como uma organização coesa onde não se perceba se a unidade é franqueada ou própria da rede, é fundamental. Nesse sistema, têm-se três atores centrais: franqueador (detentor da marca e dos procedimentos da rede), franqueado (que pode possuir uma ou várias unidades sujeitas às regras da franqueadora) e consumidor final, usuário destes produtos e serviços (Baker \& Dant, 2008). Tal configuração elenca dois "clientes" do franqueador: o franqueado e o consumidor final. $\mathrm{O}$ franqueado, atual ou futuro, demanda atenção na compra ou manutenção de unidades, e o cliente final é o foco quando se pensa em produtos ou serviços oferecidos nas unidades.

O franqueador impõe os seus próprios padrões na rede de franchising. A perfeita coordenação depende de uma série de fatores associados, entre os quais temos: marca, modelo de negócio testado, treinamento, manuais e controle da rede (Caves \& Murphy II, 1976; Toledo \& Proença, 2005). Além desses aspectos operacionais contínuos, dois dos aspectos estratégicos deste negócio incluem a escolha, por parte do franqueador, de quem serão os franqueados (Jambulingam \& Nevin, 1999), e o atendimento à legislação de franchising em vigor no Brasil, como, por exemplo, a elaboração de documento prévio ao início da parceria (Bitti, Aquino \& Amato Neto, 2013). Esses pontos influenciam diretamente a imagem que a franquia irá transmitir ao mercado. Para Elango e Fried (1997), a propriedade do sistema de franchising está dividida entre o franqueador e o franqueado, porém o primeiro tem um controle sobre toda a rede que o segundo só consegue exercer em sua unidade com restrições contratuais.

A busca de coordenação do sistema de franquia sugere que o franqueador deva buscar, entre suas metas e as do franqueado, a preservação e valorização da marca - ativo que ambos compartilham (Vance, Fávero \& Luppe, 2008). Padronizar o domínio de seu negócio requer que o franqueador possa: capacitar os franqueados; coletar, processar, analisar, e difundir conhecimento na rede; estabelecer normas, processos, políticas e padrões (Cherto, Campora \& Garcia, 2006). Além disso, a cultura organizacional da franqueadora permeia toda a rede. Mesmo estando em 
locais diferentes, o sistema de franchising busca a padronização, mesmo que em alguns mercados haja uma adaptação mínima a padrões locais (Bodey, Shao \& Ross, 2017). Nesse contexto, é importante a transferência de padrões operacionais e de valores, levando-se em conta a desconcentração das unidades (separação operacional e geográfica). A imposição de padrões e a ampla difusão de símbolos e significados induzem a formação de uma cultura organizacional coesa em todas as unidades (Matos \& Lebarcky, 2011; Coughlan, Anderson, Stern \& El-Ansary, 2002).

A Figura 1 traz uma representação da estrutura da franquia, em que os consumidores devem enxergar a organização como um elemento estrategicamente estável. Desta perspectiva percebe-se que as unidades devem ser percebidas como partes "iguais" da organização, mantendo o core business e a experiência do cliente homogêneos ao longo do conjunto de unidades, sejam elas franqueadas ou próprias (Azevedo \& Silva, 2001). As unidades, sem exceção, sejam próprias ou franqueadas, têm o intuito de ser reflexo do que o consumidor espera da franqueadora. Tal sentimento permeia não só os consumidores finais como também novos franqueados, afinal estar associado a uma marca já conceituada proporciona ganhos de reputação inexistentes em negócios emergentes (Sardy \& Alon, 2007).

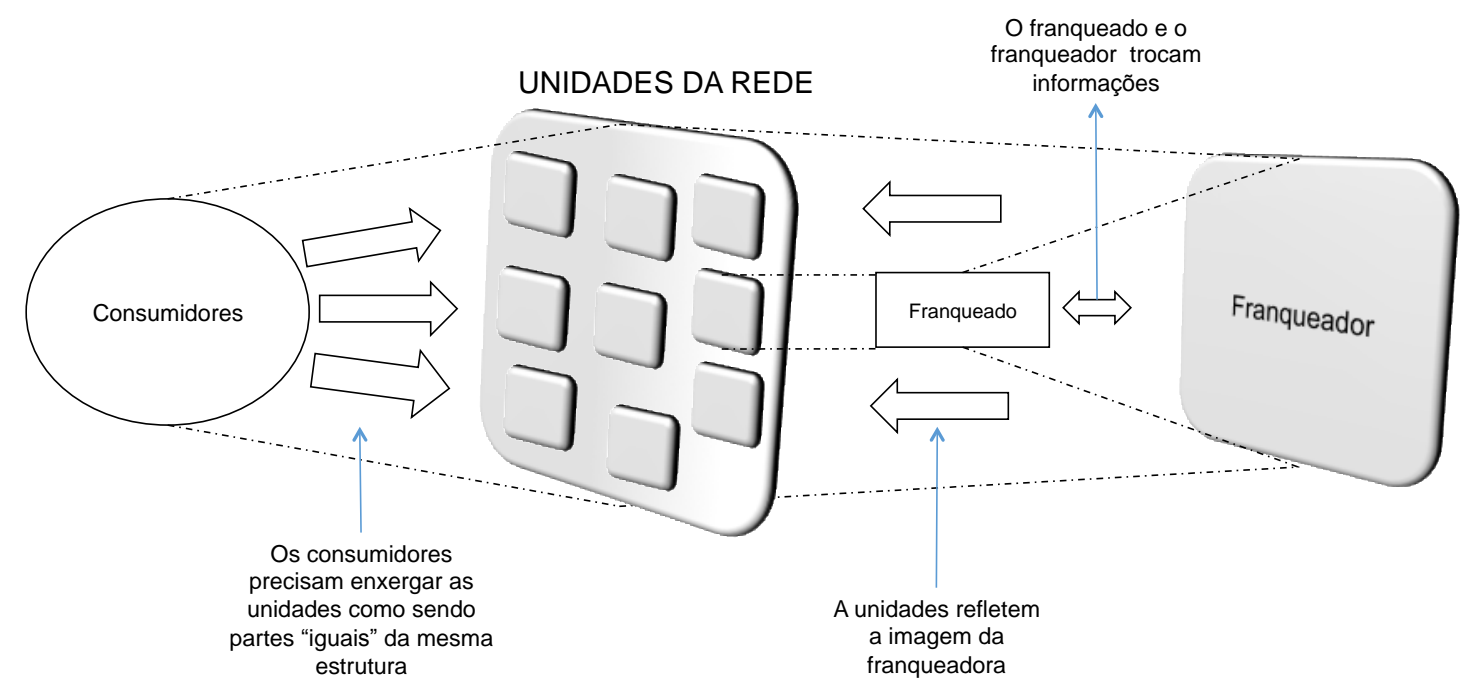

Figura 1 - Unidades franqueadas como reflexo do franqueador

Fonte: Elaboração própria

A construção da marca passa, assim, por estratégias diversas; a abordada nessa pesquisa passa pela concepção de Bansal e Roth (2000), que sinaliza que a preocupação de uma pessoa com o meio ambiente se reflete em seus valores e influencia suas ações e atitudes. Nesta perspectiva, as empresas podem adotar uma abordagem mais voltada a sinalizar sua preocupação ambiental e sociorresponsável que pode gerar ganhos, como apontados por Luo e Bhattacharya (2006).

Uma rede de franquia atinge sempre uma proporção em escala maior quando comparada com empresas individuais. Isso se dá porque as marcas de franquias possuem um número maior de estabelecimentos operando segundo seus preceitos e atingem uma abrangência maior no mercado (Tassigny, Silva, Brasil, Bizarria \& Frota 2015). Assim, uma rede de franquias que se propõe ser sustentável e com uma abordagem com foco no aspecto ambiental deve transmitir esta mensagem para o mercado e disseminar tais conceitos entre seus franqueados a ponto, inclusive, de se fazer um uso competitivo desta imagem, conforme explorado na sessão seguinte.

\section{DIMENSÃO AMBIENTAL E COMPETITIVIDADE EMPRESARIAL}

Segundo Barney (1986), as empresas que adotam posturas convergentes com determinados posicionamentos podem transformá-los em vantagem competitiva sustentável e até mesmo obter 
desempenho financeiro superior. Para este autor, cultura organizacional normalmente é definida como um conjunto complexo de valores, crenças, suposições e símbolos que definem a maneira pela qual uma empresa conduz seus negócios. Um dos aspectos mais relevantes em termos de cultura organizacional na atualidade diz respeito ao posicionamento organizacional quanto a questões de ordem ambiental (Albertini, 2013). Neste sentido, Elkington (1997) enfatiza os riscos crescentes que estão associados a um comportamento "incorreto" por parte das organizações, podendo acarretar danos à reputação das firmas. A não obediência às regulações ambientais é outra ameaça à imagem das empresas e saúde dos negócios, podendo ter fortes impactos na sua competitividade (Porter \& Linde, 1995; Nidumolu, Prahalad \& Rangaswami, 2009)

Dentre os elementos deste debate, está a importância e o diferencial que significa vincular a imagem da empresa aos valores ambientais, sendo que, conforme pontuam Nidumolu et al. (2009, p.1), “[...] não há alternativas ao desenvolvimento sustentável”. Não obstante, por mais que se argumente que as questões ambientais podem vir a ser uma oportunidade para empresas ganharem competitividade e se diferenciarem no mercado, investimentos nesta área ainda representam pequena parcela dos gastos, posição que é sustentada por uma visão estática das organizações, com foco em custos operacionais. Soma-se a esta posição uma ausência de indicadores mais objetivos que evidenciem uma relação positiva entre gastos na dimensão ambiental e maior competitividade.

Um ponto essencial nesta discussão está relacionado à presença de assimetria de informação em interações entre agentes envolvidos em trocas comerciais. O fenômeno da assimetria de informação é representado pela situação na qual o ofertante detém um conhecimento em maior profundidade sobre a qualidade do bem negociado do que o demandante (Pindyck \& Rubinfeld, 1994; Mankiw, 2005). Dentre os principais fatores resultantes deste fenômeno, expõem-se a seleção adversa e o risco moral que representam fatores de potencial alteração do equilíbrio natural dos mercados.

Seleção adversa trata da situação na qual os fornecedores de bens de alta qualidade não são capazes (ou não têm incentivos) de oferecer informações sobre este bem para o mercado, promovendo a tendência de domínio mercadológico dos bens de baixa qualidade (sendo o preço o único mecanismo de informação), assim, criando um desvio na eficiência do mercado. Por sua vez, risco moral faz referência ao comportamento do agente que, impossibilitado seu monitoramento, age de forma distinta daquela prevista, gerando um caso de ação oculta (Pindyck \& Rubinfeld, 1994; Varian, 1996; Mankiw, 2005).

Uma ferramenta de correção destas falhas de mercado é o uso de instrumentos de sinalização. A sinalização é o meio pelo qual ofertantes enviam informações para o mercado a respeito dos atributos dos produtos/serviços que comercializam (Spence, 1973). Dentre os principais mecanismos de sinalização, se evidenciam as certificações, garantias, campanhas publicitárias, marcas e a propaganda (Fischer \& Waquil, 2009). Assim, definem-se sinais como aquelas informações que um agente pode adquirir e manipular e que são enviadas para o mercado com a finalidade de reduzir a assimetria de informação entre as partes, ajustando a interação dos mercados (Spence, 1973).

Para o ambiente específico de gestão ambientalmente responsável, Schaltegger (1997) utiliza a abordagem de economia da informação como elemento analítico de instrumentos de sinalização. De acordo com este autor, são os benefícios de mercado (externos) que geram incentivos para a emissão de sinais por parte das organizações ${ }^{1}$. Endrikat et al. (2014) identificam que tais ganhos estariam principalmente atrelados às melhorias na reputação organizacional.

Alguns avanços nesta direção incluem estudos acerca da adoção do ISO 14.000, norma esta que certifica práticas de sustentabilidade ambiental nas empresas. Nessas análises, o desempenho financeiro das empresas é comparado a empresas que não possuem a certificação. Alguns estudos afirmam que a adoção da ISO 14.000 pode melhorar o desempenho das empresas e seria um ponto positivo, ou seja, sinônimo de resultado superior (Campos et al., 2009; Lo, Yeung \& Cheng, 2012; Thakore, Lowe \& Nicholls, 2012; Wiengarten \& Lam, 2017); outros indicam que empresas certificadas são mais propensas a terem resultados superiores (Heras-Saizarbitoria, Molina-Azorín 
\& Dick, 2011); há ainda estudos que argumentam que ter ISO 14.000 não é algo relevante para os investidores (Alberton \& Costa Jr, 2007).

Ainda que se identifique um predomínio de associações positivas entre ganhos mercadológicos e financeiros e a adoção de práticas ambientalmente sustentáveis, a literatura empírica sobre o tema apresenta resultados contraditórios (Albertini, 2013; Endrikat et al., 2014). Explicações para esta situação dizem respeito à qualidade e relevância percebida por estes sinais como elementos diferenciadores entre os agentes econômicos (Fischer \& Waquil, 2009; Hahn \& Kühnen, 2013; Schaltegger, 1997). Neste sentido, e de acordo com as proposições teóricas da economia da informação (Spence, 1973), a emissão de sinais mercadológicos deve ser percebida como agregadora de valor e capaz de gerar distinção entre os agentes envolvidos.

Em face destas observações teóricas e empíricas, propõem-se a hipótese e sub-hipóteses a serem testadas nesta pesquisa:

H1. Franquias envolvidas em ações de gestão ambiental sustentável aptas a serem sinalizadas para o mercado estão associadas a um desempenho financeiro corporativo superior em relação àquelas que não adotam tais expedientes.

Hla. Franquias envolvidas em ações de gestão ambiental sustentável aptas a serem sinalizadas para o mercado estão associadas a uma maior lucratividade em relação àquelas que não adotam tais expedientes.

H1b. Franquias envolvidas em ações de gestão ambiental sustentável aptas a serem sinalizadas para o mercado estão associadas a um maior faturamento em relação àquelas que não adotam tais expedientes.

H1c. Franquias envolvidas em ações de gestão ambiental sustentável aptas a serem sinalizadas para o mercado estão associadas a um menor tempo de retorno do investimento realizado em relação àquelas que não adotam tais expedientes.

Não obstante, há alguns aspectos a serem considerados na apropriação de tais hipóteses. Primeiramente, vale a pena ressaltar que o ambiente macroeconômico desempenha um papel importante nesta discussão. Pesquisas anteriores apontam uma maior efetividade dos mecanismos de sinalização ambiental em países desenvolvidos, o que é atribuído ao fato de que alto desempenho ambiental e de qualidade dos produtos/serviços são sinalizados parcialmente por meio de preços maiores quando o custo de produção destes produtos for maior (Mahenc, 2008). Desta forma, a relevância dos atributos de qualidade será maior em países de maior poder aquisitivo nos quais os compradores estão não apenas dispostos, mas também aptos a pagar um preço-prêmio por produtos diferenciados (Auriol \& Schilizzi, 2003).

Uma última discussão diz respeito ao prazo dos retornos oriundos da prática de sinalização: Corbett, Montes-Sancho e Kirsch (2005) demonstram que a emissão de sinais para o mercado resulta em desempenhos financeiros superiores às suas concorrentes somente três anos após a implementação de mecanismos de certificação ${ }^{2}$. Estas ponderações acerca da hipótese proposta são fundamentais para a interpretação dos resultados empíricos desta pesquisa.

\section{ABORDAGEM METODOLÓGICA}

A estratégia metodológica adotada tem em conta o objetivo de verificar associações entre resultados corporativos e o envolvimento de franquias em ações de gestão ambiental passíveis de serem sinalizadas para o mercado. A abordagem dos dados empíricos contemplados nessa pesquisa, tendo em vista as hipóteses apresentadas, está fundamentada em informações e estimativas de empresas envolvidas com atividades de franquia durante os anos 2013-2014.

Em termos analíticos, a composição das variáveis de interesse é apresentada na figura 1. Seguindo a lógica subjacente ao exercício de pesquisa contido nesse artigo, o construto de central interesse faz referência às variáveis Sust_AMB1 e Sust_AMB2, as quais identificam (variáveis 
binárias) empresas que tenham desenvolvido estratégias voltadas para a realização de projetos de sustentabilidade ambiental. Mais especificamente, Sust_AMB1 faz referência a programas e estratégias próprias das empresas em torno de atividades de sustentabilidade ambiental. Incluem-se, neste quesito, atividades explicitamente vinculadas à imagem da empresa, sendo informadas por meio de programas específicos e inclusão da sustentabilidade ambiental como missão ou valores da empresa. O ponto mais relevante, neste quesito, trata da atuação ambientalmente sustentável e concomitante comunicação clara para o mercado acerca destas atividades. Tal abordagem segue as indicações de Schultze e Trommer (2012), os quais identificam que a participação voluntária em atividades e propostas relacionadas à gestão ambiental funciona como um indicador de sustentabilidade ambiental da organização.

Contudo, esta variável não determina vinculação obrigatória a certificações outorgadas por órgãos independentes. Com base na expectativa de que tais elementos de sinalização possam emitir informações pouco valiosas ao mercado (dada a sua possível vinculação a riscos morais) (Ann, Zailani \& Wahid, 2006; Van Kooten, Nelson \& Vertinsky, 2005), estimações complementares são conduzidas pela variável Sust_AMB2, a qual identifica as franquias que estão vinculadas a certificações outorgadas por órgãos independentes. Para esta variável, são identificadas franquias vinculadas a programas da FSC, SQS Sindilav Aneel, Green Building Council, Destaque Sustentabilidade Afras, Prêmio Eco AmCham, Prêmio Valor Social (o qual inclui valores ambientais), ABF Franchising de Baixo Carbono e Certificado Empresa Sustentável. Estes dados foram obtidos por meio de exaustivas pesquisas em websites e documentos da base empresarial de franquias disponibilizada pela $\mathrm{ABF}^{3}$.

Com base nesta proposta, os construtos independentes na etapa de modelagem abordam impactos da variável estatística conjunta sobre faturamento (FAT), lucratividade (LUC) e prazo de retorno de investimentos (RET_MS), assim propondo uma abordagem tridimensional da perspectiva de desempenho da amostra de franquias analisadas. Com vistas ao desenvolvimento de modelos de regressão, apresentam-se as variáveis de controle da etapa empírica. INV_Inic e TX_FRA oferecem elementos que, uma vez inseridos na etapa de modelagem, mantêm constantes os resultados em termos de comprometimento financeiro inicial para cada franquia. Em sentido complementar, a inclusão de CAP_Giro, Area, Funcs e ROY permite o controle de elementos de ordem operacional dos empreendimentos. A inserção destas seis variáveis-controle, assim, permite analisar os efeitos líquidos das estratégias ambientais (e sua respectiva sinalização) mantendo-se constantes questões relacionadas, em sentido amplo, às características operacionais e de tamanho das franquias analisadas sobre os construtos dependentes (FAT, LUC e RET_MS). Tendo-se em conta que o espectro amostral é restrito a franquias incluídas no macrossetor de serviços, indicadores setoriais não são incluídos na análise.

\begin{tabular}{|c|c|c|}
\hline Variável & Descrição & Fonte \\
\hline Sust_AMB1 & $\begin{array}{l}\text { Adoção de estratégias e programas de sustentabilidade ambiental explicitamente } \\
\text { vinculadas à atuação da empresa, incluindo-se ou não participação em } \\
\text { certificações outorgadas por terceiros. Toma-se este indicador como variável } \\
\text { binária ("1" em caso de adoção, "0" em caso contrário), funcionando como } \\
\text { indicador do engajamento corporativo com atividades de responsabilidade } \\
\text { ambiental. }\end{array}$ & $\begin{array}{l}\text { Websites } \\
\text { institucionais e } \\
\text { documentos } \\
\text { das franquias }\end{array}$ \\
\hline Sust_AMB2 & $\begin{array}{l}\text { Adoção de certificações e participação em programas de sustentabilidade } \\
\text { ambiental outorgados por órgãos independentes. Estão excluídos desta variável, } \\
\text { a gestão de programas de sustentabilidade ambiental sem chancelas externas. } \\
\text { Toma-se este indicador como variável binária ("1" em caso de adoção, "0" em } \\
\text { caso contrário), funcionando como indicador do engajamento corporativo com } \\
\text { atividades de responsabilidade ambiental. }\end{array}$ & $\begin{array}{l}\text { Websites } \\
\text { institucionais e } \\
\text { documentos } \\
\text { das franquias }\end{array}$ \\
\hline INV_Inic & $\begin{array}{l}\text { Investimento inicial: o investimento inicial se refere às instalações, comunicação } \\
\text { visual, equipamentos utilizados no negócio, licença de programas de controle, } \\
\text { estoque inicial, entre outros. Também pode ter uma parte utilizada para a reforma } \\
\text { do ponto e possíveis ajustes. }\end{array}$ & $\mathrm{ABF} / \mathrm{Afras}$ \\
\hline
\end{tabular}


Taxa de franquia: a taxa de franquia ou taxa de adesão ao sistema, é o pagamento feito no instante em que o novo franqueado adere aos termos e negócios da franquia. Nesse valor, estão inclusas as despesas com os serviços que serão TX_FRA prestados antes da abertura do negócio. Essa taxa contempla os treinamentos,

$\mathrm{ABF} / \mathrm{Afras}$ orientação para escolha do ponto comercial, projetos arquitetônicos, por exemplo, e varia de empresa para empresa. Seu valor geralmente está atrelado ao reconhecimento da marca no mercado ou ao prazo de contrato.

Capital de Giro: o valor necessário para se operar o negócio. É, portanto, o ativo

CAP_Giro circulante que sustenta as operações do dia-a-dia da unidade franquiada e representa a parcela do investimento que circula durante a condução normal dos

ABF/Afras negócios.

\begin{tabular}{|c|c|c|}
\hline Area & Área Mínima M²: área mínima interna da unidade. & $\mathrm{ABF} / \mathrm{Afras}$ \\
\hline Funcs & № Funcionários: número de funcionários para a operação padrão do negócio. & $\mathrm{ABF} / \mathrm{Afras}$ \\
\hline ROY & $\begin{array}{l}\text { Royalties: A palavra royalty, de origem inglesa, designa a importância paga pelo } \\
\text { uso da marca, patente e outros bens, dando o direito de ser usado por terceiros. } \\
\text { A taxa de royalties se refere ao valor pago pelo uso da marca do franqueador } \\
\text { pelo franqueado. Essa taxa, geralmente mensal, é paga levando-se em conta um } \\
\text { percentual de faturamento ou de compras feitos junto ao franqueador que } \\
\text { também fornece produtos. }\end{array}$ & $\mathrm{ABF} / \mathrm{Afras}$ \\
\hline FAT & $\begin{array}{l}\text { FB Médio Mensal: Faturamento bruto mensal é o faturamento da unidade médio } \\
\text { em um ano fiscal sem descontar as taxas, impostos, aluguel e outras despesas } \\
\text { acessórias do negócio. }\end{array}$ & $\mathrm{ABF} / \mathrm{Afras}$ \\
\hline LUC & $\begin{array}{l}\text { Lucro Médio Mensal: Baseado no lucro médio de um ano fiscal, tanto o lucro } \\
\text { médio mensal como o faturamento bruto mensal podem variar se analisados mês } \\
\text { a mês devido a sazonalidade de algumas atividades. }\end{array}$ & $\mathrm{ABF} / \mathrm{Afras}$ \\
\hline RET_MS & $\begin{array}{l}\text { Prazo de Retorno (meses): O prazo de retorno ou payback é o tempo decorrido } \\
\text { entre a abertura da unidade e o momento no qual o lucro apurado se iguala ao } \\
\text { valor inicial gasto para abrir a unidade (instalação da empresa, capital de giro e } \\
\text { taxa de franquia). }\end{array}$ & $\mathrm{ABF} / \mathrm{Afras}$ \\
\hline
\end{tabular}

Figura 2. Descrição das variáveis da amostra

Fonte: Os autores

Seguindo as exposições realizadas, a estrutura básica dos modelos regressivos segue a proposta contida na equação 1 :

$$
Y_{i}=\alpha+\beta_{1} S_{i}+\beta_{2} T_{i}+\beta_{3} O_{i}+\mu_{i}
$$

Tem-se que $\mathbf{Y}$ é um indicador de output válido para os três construtos independentes (FAT, LUC e RET_MS), $\boldsymbol{\alpha}$ é a constante do modelo, $\boldsymbol{\beta}_{\mathbf{k}}$ são os coeficientes de cada uma das dimensões dependentes, S representa os marcadores binário Sust_AMB1 e SUT_AMB2, T é uma dimensão de tamanho composta por Invic_Inic e TX_FRA (Binvic_Inic $\left.+\beta T X \_F R A\right)$, O é uma dimensão de aspectos operacionais composta por CAP_Giro, Area, Funcs e ROY $\left(\beta c a p \_\right.$Giro + Barea $+\beta f u n c s$ $+\beta r o y), \boldsymbol{\mu}$ é o termo de erro (resíduo) do modelo e i identifica cada rede de franquia. A relevância do controle por tamanho organizacional e por aspectos operacionais segue a estrutura analítica proposta por (Endrikat et al., 2014).

Foram tomados logaritmos das variáveis com vistas a suavizar a distribuição da amostra para cada uma das dimensões de análise, assim homogeneizando a interpretação dos resultados para uma lógica de elasticidades. Tal procedimento não foi aplicado à variável Sust_ABF por se tratar de um marcador binário (0 ou 1). Devido à identificação de indícios de violação da hipótese de homoscedasticidade dos resíduos, procedemos à aplicação de regressões com heteroscedasticidade corrigida. Este método envolve (i) uma estimação por MQO do modelo de interesse, seguido de (ii) uma regressão auxiliar que gera estimativas do termo de erro, e (iii) aplicação de mínimos quadrados ponderados, utilizando, como fator de ponderação, o elemento recíproco da variância estimada. Na regressão auxiliar são regredidos os logs dos resíduos ao quadrado da estimação MQO sobre os regressores originais e suas representações quadráticas. A transformação em logs garante que as variâncias estimadas não assumam valores negativos. Por último, com vistas à obtenção de 
uma verificação adicional dos resultados e de suas implicações, são realizadas estimações com a exclusão de quiosques de franquias, mantendo-se somente dados de lojas-padrão ${ }^{4}$.

\section{RESULTADOS}

Inicialmente, apresenta-se uma descrição da amostra para as variáveis analíticas (Tabela 1). Uma primeira avaliação do comportamento destes vetores permite o entendimento da importância da aplicação de modelos regressivos multivariados, uma vez que se percebem - para todos os casos - padrões de intensa heterogeneidade nos construtos de pesquisa. Tais características demonstram alto nível de correspondência para a análise da amostra completa e para o caso em que são excluídas observações de quiosques ou outras unidades alternativas de instalação de franquias (mantendo-se somente as lojas-padrão).

Também, é importante notar os padrões de concentração dos construtos Sust_AMB1 e Sust_AMB2. Se, por um lado, a adoção de estratégias e iniciativas próprias das empresas (Sust_AMB1) têm uma incidência significativa (em torno a 21\% para ambas amostras), a adoção de certificações de sustentabilidade ambiental outorgadas por órgãos independentes (Sust_AMB2) é extremamente concentrada em poucas observações (ligeiramente superior a $3 \%$ dos casos), identificando a baixa propensão de franquias no Brasil de aderirem a estes instrumentos de certificação. Tal comportamento dos dados deverá ser levado em conta como fator indutor de sensibilidade nos exercícios de regressão. Vale a pena refletir e sugerir, como linha futura de pesquisa, se o padrão setorial de tais franquias (macrossetor de serviços) apresenta um comportamento diferenciado em relação a outras atividades econômicas ou se a realidade empresarial brasileira está adequadamente representada por esta amostra. Os resultados deste exercício, espera-se, deverão oferecer insights sobre este fenômeno a partir da tentativa de identificação de relacionamento entre estes instrumentos e resultados empresariais.

Tabela 1.

Descrição das variáveis da amostra

\begin{tabular}{|c|c|c|c|c|c|c|}
\hline & \multicolumn{2}{|c|}{ Média } & \multicolumn{2}{|c|}{ Desvio Padrão } & \multicolumn{2}{|c|}{ Coeficiente de Variação } \\
\hline & $\begin{array}{l}\text { Amostra } \\
\text { Completa } \\
\end{array}$ & $\begin{array}{c}\text { Somente Lojas- } \\
\text { Padrão }\end{array}$ & $\begin{array}{l}\text { Amostra } \\
\text { Completa } \\
\end{array}$ & $\begin{array}{c}\text { Somente } \\
\text { Lojas-Padrão }\end{array}$ & $\begin{array}{l}\text { Amostra } \\
\text { Completa }\end{array}$ & $\begin{array}{c}\text { Somente } \\
\text { Lojas-Padrão }\end{array}$ \\
\hline INV_INIC & $216.754,62$ & $232.645,20$ & $197.006,61$ & $204.322,89$ & 0,91 & 0,88 \\
\hline TX_FRA & $43.431,00$ & $45.511,46$ & $41.389,84$ & $43.848,46$ & 0,95 & 0,96 \\
\hline CAP_GIRO & $34.855,13$ & $35.798,92$ & $29.660,19$ & $28.585,05$ & 0,85 & 0,80 \\
\hline ROY & 0,07 & 0,07 & 0,09 & 0,09 & 1,29 & 1,31 \\
\hline AREA & 72,75 & 78,57 & 76,70 & 78,62 & 1,05 & 1,00 \\
\hline FUNCS & 18,95 & 21,14 & 187,50 & 202,30 & 9,89 & 9,57 \\
\hline FAT & $78.923,81$ & $82.515,36$ & $58.378,43$ & $60.574,41$ & 0,74 & 0,73 \\
\hline LUC & 0,25 & 0,25 & 0,11 & 0,12 & 0,47 & 0,48 \\
\hline \multirow[t]{3}{*}{ RET_MS } & 27,06 & 26,95 & 9,15 & 9,24 & 0,34 & 0,34 \\
\hline & \multicolumn{4}{|c|}{ Frequência } & & \\
\hline & \multicolumn{2}{|c|}{ Amostra Completa } & \multicolumn{2}{|c|}{ Somente Lojas-Padrão } & & \\
\hline SUST_AMB1 & \multicolumn{2}{|c|}{53 incidências $(20,8 \%)$} & \multicolumn{2}{|c|}{46 incidências $(21 \%)$} & & \\
\hline SUST_AMB2 & \multicolumn{2}{|c|}{8 incidências $(3,1 \%)$} & \multicolumn{2}{|c|}{8 incidências $(3,7 \%)$} & & \\
\hline
\end{tabular}

Fonte: Os autores

O próximo passo da análise consiste na avaliação dos resultados da estimação dos modelos regressivos de acordo com a estrutura previamente apresentada na seção metodológica. Primeiramente, a análise dos resultados das estimações dos modelos de regressão (Tabela 2) possui foco na variável Sust_AMB1 para as diferentes dimensões dependentes e para os dois cortes 
amostrais utilizados. Uma avaliação do poder explanatório dos modelos (a partir dos dados de $\mathrm{R}^{2}$ ) permite identificar um grau satisfatório de adequação dos modelos, especialmente para as estimações de FAT e RET_MS. Em termos da verificação da variável Sust_AMB1, os achados não permitem qualquer tipo de identificação de relações positivas entre este construto de interesse e as variáveis dependentes.

Apesar da significância estatística ser observada nas estimações para RET_MS, é importante ter em conta que nos modelos de RET_MS, sinais positivos estão associados a tempos maiores de recuperação do investimento realizado, ou seja, piores resultados operacionais de curto prazo. Isto ocorre mesmo com o controle da variável INV_INIC, isto é, os comprometimentos financeiros iniciais que são compreendidos na abertura do negócio. Por si, os gastos relacionados a atividades vinculadas à sustentabilidade ambiental também correspondem a investimentos realizados pelas empresas. Ademais, percebe-se ampla correspondência entre os resultados observados para a amostra completa e para o corte que contempla somente lojas-padrão, o que está de acordo com as estatísticas descritivas apresentadas anteriormente. Estes resultados iniciais sugerem a rejeição das hipóteses propostas para o caso de sinais "próprios" (não outorgados por órgãos independentes), demonstrando sua incapacidade de gerar valor financeiro para as franquias em um horizonte temporal de curto prazo.

Com respeito às variáveis-controle, alguns breves apontamentos merecem destaque. As variáveis de "tamanho" (INV_Inic e TX_FRA) possuem baixa associação positiva com os resultados financeiros obtidos, o que é contrário à expectativa quanto a estes itens. Quanto às variáveis de ordem operacional (CAP_Giro, Area, Funcs e ROY), evidências negativas se limitam aos valores percentuais de royalties envolvidos no contrato de franquia. Por sua vez, a área de atendimento e o número de funcionários envolvidos representam maiores possibilidades de lucratividade e faturamento, respectivamente.

Tabela 2.

Estimação dos modelos de regressão com heteroscedasticidade corrigida - Variável Sust_AMB1

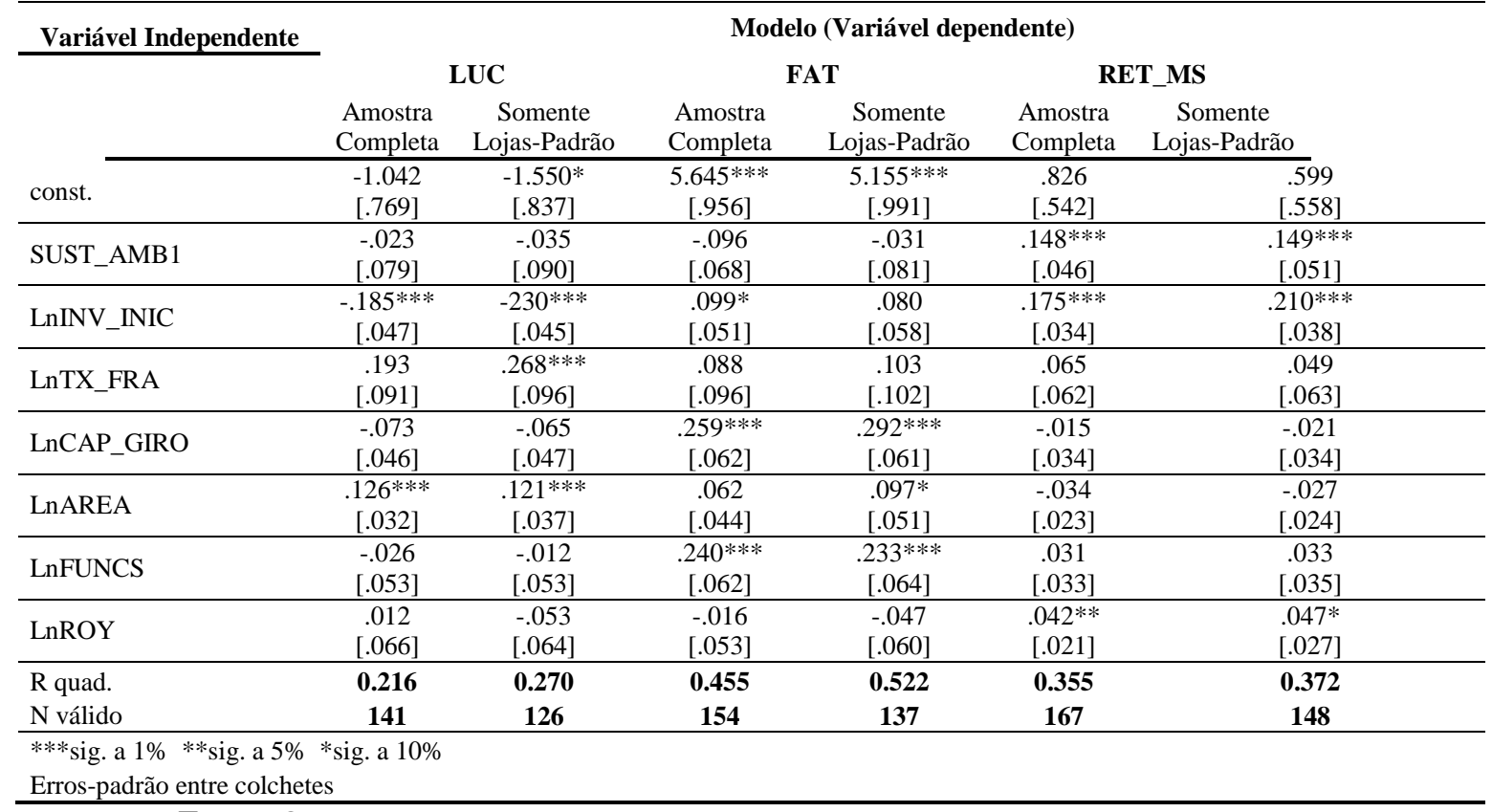

Fonte: Os autores

Em um segundo momento, repetimos as análises expostas na Tabela 2 com a substituição da variável Sust_AMB1 por Sust_AMB2, isto é, considerando-se somente aqueles casos de franquias vinculadas a programas de certificação outorgados por órgãos independentes. Conforme demonstrado nas estatísticas descritivas, Sust_AMB2 encontra apenas oito incidências nas amostras 
utilizadas, o que pode gerar um indesejável nível de sensibilidade nos modelos econométricos. Assim, resultados desta variável deverão ser vistos como sugestivos e não como conclusivos. Por outro lado, a baixa taxa de adesão a estes instrumentos de gestão e sinalização traz informações relevantes sobre a percepção dos empresários brasileiros quanto a tais práticas mercadológicas.

Tabela 3.

Estimação dos modelos de regressão com heteroscedasticidade corrigida - Variável Sust_AMB2

\begin{tabular}{|c|c|c|c|c|c|c|}
\hline \multirow[t]{3}{*}{ Variável Independente } & \multicolumn{6}{|c|}{ Modelo (Variável dependente) } \\
\hline & \multicolumn{2}{|c|}{$\mathbf{L U C}$} & \multicolumn{2}{|c|}{ FAT } & \multicolumn{2}{|c|}{ RET_MS } \\
\hline & $\begin{array}{c}\text { Amostra } \\
\text { Completa }\end{array}$ & $\begin{array}{c}\text { Somente } \\
\text { Lojas-Padrão }\end{array}$ & $\begin{array}{c}\text { Amostra } \\
\text { Completa } \\
\end{array}$ & $\begin{array}{c}\text { Somente } \\
\text { Lojas-Padrão }\end{array}$ & $\begin{array}{c}\text { Amostra } \\
\text { Completa }\end{array}$ & $\begin{array}{c}\text { Somente } \\
\text { Lojas-Padrão }\end{array}$ \\
\hline const. & $\begin{array}{c}-1.369^{*} \\
{[.790]}\end{array}$ & $\begin{array}{c}-1.926^{* * *} \\
{[.873]}\end{array}$ & $\begin{array}{c}5.825 * * * \\
{[.964]}\end{array}$ & $\begin{array}{c}5.297 * * * \\
{[.982]}\end{array}$ & $\begin{array}{c}.593 \\
{[.550]}\end{array}$ & $\begin{array}{c}.353 \\
{[.581]} \\
\end{array}$ \\
\hline SUST_AMB2 & $\begin{array}{c}-.161 \\
{[.189]} \\
\end{array}$ & $\begin{array}{l}-.146 \\
{[.210]} \\
\end{array}$ & $\begin{array}{c}.008 \\
{[.419]} \\
\end{array}$ & $\begin{array}{l}-.007 \\
{[.384]} \\
\end{array}$ & $\begin{array}{l}.284 * * * \\
{[.090]}\end{array}$ & $\begin{array}{l}.316^{* * * *} \\
{[.095]}\end{array}$ \\
\hline LnINV_INIC & $\begin{array}{c}-.150 * * * \\
{[.047]} \\
\end{array}$ & $\begin{array}{c}-.203 * * * \\
{[.045]}\end{array}$ & $\begin{array}{l}.127 * * \\
{[.056]}\end{array}$ & $\begin{array}{c}.098 \\
{[.061]}\end{array}$ & $\begin{array}{c}.176^{* * * *} \\
{[.034]}\end{array}$ & $\begin{array}{c}.195 * * * \\
{[.036]}\end{array}$ \\
\hline LnTX_FRA & $\begin{array}{l}.180 * \\
{[.094]}\end{array}$ & $\begin{array}{c}.278^{* * * *} \\
{[.106]}\end{array}$ & $\begin{array}{c}.012 \\
{[.102]}\end{array}$ & $\begin{array}{c}.066 \\
{[.103]}\end{array}$ & $\begin{array}{c}.088 \\
{[.062]}\end{array}$ & $\begin{array}{c}.090 \\
{[.064]}\end{array}$ \\
\hline LnCAP_GIRO & $\begin{array}{c}-.068 \\
{[.048]} \\
\end{array}$ & $\begin{array}{c}-.062 \\
{[.046]} \\
\end{array}$ & $\begin{array}{c}.263 * * * \\
{[.060]} \\
\end{array}$ & $\begin{array}{c}.286 * * * \\
{[.060]} \\
\end{array}$ & $\begin{array}{l}-.009 \\
{[.034]} \\
\end{array}$ & $\begin{array}{c}-.013 \\
{[.034]} \\
\end{array}$ \\
\hline LnAREA & $\begin{array}{c}.147 * * * \\
{[.033]}\end{array}$ & $\begin{array}{c}.109 * * * \\
{[.038]}\end{array}$ & $\begin{array}{l}.095 * * \\
{[.046]}\end{array}$ & $\begin{array}{l}.115^{* * *} \\
{[.051]}\end{array}$ & $\begin{array}{l}-.046^{*} \\
{[.026]}\end{array}$ & $\begin{array}{c}-.043 \\
{[.028]}\end{array}$ \\
\hline LnFUNCS & $\begin{array}{c}-.078 \\
{[.049]} \\
\end{array}$ & $\begin{array}{c}-.043 \\
{[.050]} \\
\end{array}$ & $\begin{array}{c}.230 * * * \\
{[.064]}\end{array}$ & $\begin{array}{c}.225 * * * \\
{[.065]}\end{array}$ & $\begin{array}{c}.047 \\
{[.035]} \\
\end{array}$ & $\begin{array}{c}.051 \\
{[.036]} \\
\end{array}$ \\
\hline LnROY & $\begin{array}{c}.008 \\
{[.062]}\end{array}$ & $\begin{array}{c}-.052 \\
{[.064]}\end{array}$ & $\begin{array}{c}-.057 \\
{[.052]}\end{array}$ & $\begin{array}{c}-.056 \\
{[.059]}\end{array}$ & $\begin{array}{l}.061 * * \\
{[.024]}\end{array}$ & $\begin{array}{l}.061 * * \\
{[.027]}\end{array}$ \\
\hline R quad. & 0.230 & 0.240 & 0.461 & 0.522 & 0.367 & 0.389 \\
\hline $\mathrm{N}$ válido & 141 & 126 & 154 & 137 & 167 & 148 \\
\hline $\begin{array}{l}* * * \text { sig. a } 1 \% * * \text { sig. a } 5 \% * \text { sig } \\
\text { Erros-padrão entre colchetes }\end{array}$ & & & & & & \\
\hline
\end{tabular}

Fonte: Os autores

Os resultados da Tabela 3 não diferem substancialmente daqueles apresentados para a avaliação da variável Sust_AMB1, sendo Sust_AMB2 significativamente associada positivamente com prazos mais longos de retorno dos investimentos realizados para a instalação de unidades das franquias. Demais características dos resultados se mantêm constantes, demonstrando certo grau de correspondência entre as estimações. A partir destas observações de caráter empírico, podemos rejeitar a validade das hipóteses propostas com base na literatura para o caso da amostra de franquias analisada. Este parece ser o caso tanto para sinais "fracos" (Sust_AMB1) como para sinais "fortes" (Sust_AMB2), não se podendo identificar, em nenhum caso, a geração de um equilíbrio separador. Discussões acerca destes achados e sua articulação com a literatura existente são apresentados na próxima seção.

\section{DISCUSSÕES}

A etapa empírica desta pesquisa abordou o questionamento referente à identificação de associação entre esforços de sinalização de gestão ambiental sustentável em franquias e desempenhos econômicos superiores. Para tanto, foram desenvolvidas três hipóteses operacionais (H1a, H1b e H1c) lidando com a relação entre lucratividade, faturamento e tempo de retorno de investimentos e o uso de sinais próprios e outorgados por terceiros para iniciativas voltadas à sustentabilidade ambiental.

Contrários às expectativas, os resultados indicam que a emissão de sinais mercadológicos independentes e certificados relativos à atuação ambiental não gerou desempenho superior nos critérios avaliados, demonstrando, inclusive, resultados inferiores em termos de tempo de retorno do investimento. Assim, fica evidenciada a rejeição das hipóteses propostas em todas as instâncias elaboradas para esta pesquisa. Com efeito, sinais significativos foram associados somente com 
tempos maiores de retorno dos investimentos realizados. Tais resultados estão alinhados com aqueles de Cohen, Fenn e Konar (1997) e de Graves e Waddock (1999), os quais apontam falta de significância na associação entre desempenho empresarial e adoção de estratégias de responsabilidade ambiental corporativa. Possíveis interpretações destes resultados são multidimensionais e estabelecem implicações para este campo de pesquisa.

Em termos gerais, podemos propor que há uma desconexão no âmbito brasileiro entre as características da demanda e a valorização dos atributos intrínsecos analisados e à qualidade do instrumento de sinalização verificado. Esta situação está em sintonia com a ideia de que o comportamento de compra dos consumidores em países de renda média apresenta reduzida propensão para valorização de atributos de sustentabilidades incorporados em produtos e serviços (Albertini, 2013; Auriol \& Schilizzi, 2003). Desta forma, a relevância dos atributos de qualidade será maior em países de maior poder aquisitivo nos quais os compradores estão não apenas dispostos, mas também aptos a pagar um preço-prêmio por produtos diferenciados (Auriol \& Schilizzi, 2003). Isto é particularmente sensível para o caso de um país de renda média, como é o caso do Brasil.

Adicionalmente, ainda que a lógica subjacente ao uso de mecanismos de sinalização seja a de recompensar os agentes pelos seus produtos/serviços diferenciados, o preço-prêmio pago por diversos mercados não compensa as perdas em produtividade que ocorrem neste expediente produtivo, assumindo-se que haja propensão a pagar este valor diferencial por parte dos consumidores. Assim, técnicas de menor preocupação com qualidade ou com impactos ambientais podem se revelar mais lucrativas, tornando-se necessários maiores incentivos no mercado para produtos certificados. Na ausência destas condições institucionais, determinadas práticas passíveis de sinalização produtiva ambientalmente amigáveis se tornam economicamente inviáveis (Pearce et al, 2003; Conceição \& Barros, 2005).

Uma segunda interpretação dos resultados leva a especular que, para o ambiente específico de franquias no Brasil, há uma falta de relevância, divulgação ou credibilidade dos instrumentos de sinalização, tornando ineficientes as ferramentas de sinalização analisadas (Ann et al, 2006; Van Kooten et al, 2005). Isto porque a questão de percepção dos stakeholders acerca da relevância do instrumento de transmissão da informação também pode representar um efeito moderador substancial (Hahn \& Kühnen, 2013). Tal situação gera um contexto em que as empresas encontram reduzidos incentivos para estabelecer um envolvimento com estratégias de operação ambientalmente sustentáveis, assim prejudicando a incorporação destes mecanismos nas práticas gerenciais. Dado que esta dinâmica é indesejável para um efetivo desenvolvimento sustentável de longo prazo do sistema econômico, nossos resultados apontam para a necessidade de se promover maiores níveis de conscientização (awareness) em torno das iniciativas empresariais vinculadas à prática de gestão ambiental e suas respectivas ferramentas de sinalização. Assim, algum grau de reversão da tendência observada na amostra estudada pode ser alcançado pelas empresas em esforços de divulgação e validação dos instrumentos de sinalização utilizados (próprios ou concedidos por terceiros), assim possibilitando que estes expedientes agreguem valor aos produtos ao disponibilizar informações relevantes para os compradores (Ann et al, 2006; Van Kooten et al, 2005).

Uma terceira linha de interpretação compreende questões relacionadas às características de temporalidade das variáveis incluídas na base de dados referente à amostra utilizada. Neste sentido, as evidências empíricas podem estar associadas a problemas de timing na avaliação devido ao uso de resultados correntes. Neste caso, não se pode excluir a possibilidade de ganhos estratégicos e de imagem que tais instrumentos de gestão ambiental e sinalização possam render às organizações no longo prazo - os quais não podem ser capturados apropriadamente pelos indicadores de curto prazo utilizados nessa análise. Nesta linha de pensamento, Corbett, Montes-Sancho e Kirsch (2005) demonstram que as empresas certificadas alcançam desempenhos financeiros superiores às suas concorrentes somente três anos após a implementação de mecanismos de certificação. 
Neste contexto, podemos identificar que a adoção de práticas corporativas de sustentabilidade ambiental parece ter sua eficiência econômica atrelada a uma série de condições da dinâmica mercadológica na qual as firmas estão inseridas. Barney (1986) propõe que o uso de determinadas estratégias convergentes com determinados posicionamentos pode gerar vantagens competitivas para as organizações. Complementarmente, Albertini (2013) e Scarpellini et. al (2017) identificaram que questões de ordem ambiental são itens essenciais para a manutenção de uma cultura organizacional competitiva, argumento que já era defendido por Elkington (1997). Não obstante, estratégias empresariais são construtos multidimensionais, os quais consideram não somente a tomada de decisão por parte da empresa, mas sua relação com os mercados e as instituições (Nag et al, 2007).

É a partir desta visão que este estudo contribui para a necessidade de um entendimento mais amplo acerca da relevância das estratégias de gestão ambiental sustentável, seus mecanismos de sinalização e a geração de vantagens competitivas para as empresas. Isto porque, em um ambiente socioeconômico que produz reduzidos incentivos para o engajamento das empresas com tais atividades, deverá moderar a ampla adoção de práticas ambientalmente sustentáveis, retardando a adequação da matriz produtiva em torno a um modelo de desenvolvimento econômico sustentável. Dentro desta questão, a valorização de mecanismos de sinalização se torna um expediente de especial interesse institucional, um aspecto que parece permanecer ausente da dinâmica de negócios das franquias brasileiras.

\section{CONSIDERAÇÕES FINAIS}

Este artigo lidou com a temática de relação entre estratégias de gestão ambientalmente sustentável (e seus respectivos mecanismos de sinalização) e desempenho econômico de franquias. Surpreendentemente, os resultados demonstram a rejeição da hipótese de que tal relação é positiva para medidas de lucratividade, faturamento e tempo de retorno dos investimentos realizados.

Uma contribuição metodológica desta abordagem diz respeito à aproximação entre análises de comportamento ambientalmente responsável pelas empresas e a relevância dos mecanismos de sinalização entendidos como uma instituição econômica. Isto é particularmente relevante no contexto de atributos intrínsecos, os quais maximizam a exposição a riscos morais.

Nesta lógica, uma implicação de central interesse da nossa abordagem para desenvolvimentos teóricos e empíricos direcionados à prática de sinalização de atributos relacionados à sustentabilidade ambiental diz respeito à necessidade de se analisar o seu acoplamento com a dinâmica de incentivos econômicos para as empresas adotarem tais expedientes.

Estratégias corporativas de sustentabilidade são frequentemente analisadas partindo do pressuposto de que ganhos associados à imagem da empresa são suficientes para justificar sua relevância. Contudo, esta é uma situação precária para as firmas se não houver a geração de retornos econômicos para as partes interessadas. Desta forma, um ambiente mercadológico carente destas condições pode mover um ciclo de retroalimentação negativa que dificultará níveis crescentes de atividades empresariais que reduzam danos ao meio-ambiente.

Tais aspectos orientam a possibilidade de pesquisas futuras, principalmente quanto a dois aspectos-chave: primeiramente, estudos que dediquem atenção a identificar a existência e características de possíveis barreiras à valorização por parte da demanda em termos de práticas ambientalmente sustentáveis (incluindo renda e conscientização acerca dos mecanismos de sinalização) no ambiente específico de franquias. Partindo de uma perspectiva da economia da informação, tais abordagens podem fornecer evidências relevantes para a gestão de selos e certificações ambientais, tanto por parte das empresas adotantes como dos órgãos certificadores. Efeitos destas questões vão além do âmbito puramente mercadológico, podendo influenciar a adoção, em maior escala, de sistemas de gestão sustentáveis no Brasil.

Entre as limitações do estudo, reside o fato de este ser um estudo embasado em curto prazo, não permitindo checar aspectos evolutivos, tendências de médio e longo prazo. Outra limitação 
pode ser observada no número de empresas estudadas, fato que se dá devido a não divulgação de dados por parte de um contingente mais amplo de franqueadoras, o que impede, inclusive, que se faça uma análise setorial deste comportamento. Talvez mais relevante, o corte amostral de franquias oferece observações fundamentalmente orientadas ao macrossetor de serviços, um aspecto que pode enviesar os resultados e torná-los pouco representativos para conclusões relacionadas a outros setores da economia - principalmente indústrias de transformação.

\section{REFERÊNCIAS}

ABF-Associação Brasileira de Franquia.(2017) Números do Franchising mostrando o desempenho do setor, 2017. Recuperado em: 11 out.2017, de: http://www.abf.com.br/numeros-do-franchising/.

Aguiar, H. D. S., Pauli, S., Yu, A. S., \& Nascimento, P. T. D. S. (2016). Modeling the new franchise creation decision: the relevance of behavioral reasons. Mackenzie Management Review, 17(5), 110-137.

Albertini, E. (2013). Does environmental management improve financial performance? A metaanalytical review. Organization \& Environment, 26(4), 431-457.

Alberton, A., \& Costa Jr, N. (2007). Meio ambiente e desempenho econômico-financeiro: benefícios dos Sistemas de Gestão Ambiental (SGAs) e o impacto da ISO 14001 nas empresas brasileiras. RAC-Eletrônica, 1(2), 153-171.

Akerlof, G. A. (1970). The market for "lemons": quality uncertainty and the market mechanism. The Quarterly Journal of Economics, 84(3), 488-500.

Ann, G., Zailani, S., \& Wahid, N. A. (2006). A study on the impact of environmental management system (EMS) certification towards firms' performance in Malaysia. Management of Environmental Quality. 17(1), 73-93.

Auriol, E., \& Schilizzi, S.G.M. (2003). Quality signaling through certification: theory and an application to agricultural seed markets. IDEI Working Papers, 165.

Azevedo, P., \& Silva, V. (2001). Franquias de alimentos e coordenação de cadeias agroindustriais : uma análise empírica. Revista de Administração, 37(1), 51-62.

Baker, B., \& Dant, R. (2008). Stable plural forms in franchise systems: an examination of the evolution of ownership redirection research. Strategy and Governance of Networks, 87-112.

Bansal, P., \& Roth, K. (2000). Why companies go green: a model of ecological responsiveness. The Academy of Management Journal, 43(4), 717-736.

Barney, J. B. (1986). Organizational culture: can it be a source of sustained competitive advantage? Academy of Management Review, 11(3), 656-665.

Bitti, E. J. S., Aquino, A. C. B., \& Amato, J. (2013). Monitoramento e coordenação em redes de franquia : dispersão geográfica e automação impactando o mix contratual Informações do Artigo. Revista de Contabilidade e Organizações, 7(18), 3-13.

Bodey, K. L., Shao, W., \& Ross, M. (2017). Localization and customer retention for franchise service systems. Services Marketing Quarterly, 38(2), 100-114.

Brandt, E. (2007). Truelo de tipologias estratégicas na arena das franquias de fast food no Brasil: Porter X Miles \& Snow X Mintzberg. Revista Eletrônica de Estratégia \& Negócios, 1 (2), 160-176.

Campos, L., Grzebieluckas, C., \& Selig, P. (2009). As empresas com certificação ISO 14001 são mais rentáveis? Uma abordagem em companhias abertas no Brasil. Read: Revista Eletrônica de Administração, 15(1), 108-131. 
Caves, R., \& Murphy II (1976). W. F. Franchising: firms markets and intangible assets. Southern Economic Journal, 42(4), 572-586.

Cherto, M., Campora, F., \& Garcia, F. (2006). Franchising: uma estratégia para expansão de negócios. São Paulo: Premier Máxima Editora.

Cohen, M., Fenn, S., \& Konar, S. (1997). Environmental and financial performance: are they related? Working Paper, Vanderbilt University.

Conceição, J., \& Barros, A. (2005). Certificação e rastreabilidade no agronegócio: instrumentos cada vez mais necessários. Texto para discussão n. 1122, Instituto de Pesquisa Econômica Aplicada, Brasília.

Corbett, C., Montes-Sancho, M., \& Kirsch, D. A. (2005) The financial impact of ISO 9000 certification in the United States: an empirical analysis. Management Science, 51(7), 1046-1059.

Coughlan, A., Anderson, E., Stern, \& El-Ansary, A. (2002). Canais de marketing e distribuição. (6 ed.). Porto Alegre: Bookman.

Dahlsrud, A. (2008). How corporate social responsibility is defined: an analysis of 37 definitions. Corporate Social Responsibility and Environmental Management, 15(1), 1-13.

Dewally, M., \& Ederington, L. (2006). Reputation, certification, warranties, and information as remedies for seller-buyer information asymmetries: lessons from the online comic book market. Journal of Business, 79(2), 693-729.

Elango, B., \& Fried, V. (1997). Franchising research: a literature review and synthesis. Journal of Small Business Management, 35(3), 68-81.

Elkington, J. (1997). Cannibals with forks - Triple bottom line of 21 st century business. Stoney Creek: New Society Publishers.

Endrikat, J., Guenther, E., \& Hoppe, H. (2014). Making sense of conflicting empirical findings: a meta-analytic review of the relationship between corporate environmental and financial performance. European Management Journal, 32(5), 735-751.

Fischer, B., \& Waquil, P. (2009). Sistemas de certificação florestal no setor brasileiro de papel e celulose: influências no desempenho exportador. Revista de Economia Agrícola, 56(2), 5-19.

Friedman, M. (1970). The social responsibility of business is to increase its profits. The New York Times Magazine, September 13.

Graves, S., \& Waddock, S. (1999). A look at the financial-social performance nexus when quality of management is held constant. International Journal of Value-Based Management, 12, 87-99.

Guenther, E., \& Hoppe, H. (2014). Merging limited perspectives: a synopsis of measurement approaches and theories of the relationship between corporate environmental an financial performance. Journal of Industrial Ecology, 18(5), 689-707.

Hahn, R., \& Kühnen, M. (2013). Determinants of sustainability reporting: a review of results, trends, theory, and opportunities in an expanding field of research. Journal of Cleaner Production, $59,5-21$.

Heras-Saizarbitoria, I., Molina-Azorín, J., \& Dick, G. (2011). ISO 14001 certification and financial performance: selection-effect versus treatment-effect. Journal of Cleaner Production, 19(1), 1-12.

Jambulingam, T., \& Nevin, J. (1999). Influence of franchisee selection criteria on outcomes desired by the franchisor. Journal of Business Venturing, 14(4), 363-395. 
Lo, C., Yeung, A., \& Cheng, T. (2012). The impact of environmental management systems on financial performance in fashion and textiles industries. International Journal of Production Economics, 135(2), 561-567.

Luo, X., \& Bhattacharya, C. B. (2006). Corporate social responsibility, customer satisfaction, and market value. Journal of Marketing, 70(4), 1-18.

Mahenc, P. (2008). Signaling the environmental performance of polluting products to green consumers. International Journal of Industrial Organization, 26, 59-68.

Mankiw, N. (2005). Princípios de microeconomia. São Paulo: Pioneira Thomson Learning.

Monge, M. E. C., \& Huerta, P. A. (2015). Lucro líquido do franqueado: um sinal para a escolha de franquia em época de crise. RAE-Revista de Administração de Empresas, 55(6), 688-698.

Murray, J. (2004). Corporate social responsibility: an overview of principles and practice. International Labour Office. Working Paper.

Nag, R., Hambrick, D., \& Chen, M. (2007). What is strategic management, really? Inductive derivation of a consensus definition of the field. Strategic Management Journal, 28(9), 935-955.

Nidumolu, R., Prahalad, C. K., \& Rangaswami, M. (2009). Why sustainability is now the key driver of innovation. Harvard Business Review, 87(9), 56-64.

Pearce, D., Putz, F., \& Vanclay, J. K. (2003). Sustainable forestry in the tropics: panacea or folly? Forest Ecology and Management, 172, 229-47.

Pindyck, R., \& Rubinfeld, D. (1994). Microeconomia. São Paulo: Makron Books.

Porter, M, \& Linde, C. (1995). Toward a new conception of the environment-competitiveness relationship. Journal of Economic Perspectives, 9(4), 97-118.

Sardy, M., \& Alon, I. (2007). Exploring the differences between franchisee entrepreneurs and nascent entrepreneurs. International Entrepreneurship and Management Journal, 3(4), 403-418.

Scarpellini, S., Valero-Gil, J., Rivera-Torres, P., \& Garcés-Ayerbe, C. (2017). Analysis of the generation of economic results in the different phases of the pro-environmental change process. Journal of Cleaner Production, 168.

Schaltegger, S. (1997) Information costs, quality of information and stakeholder involvement: the necessity of international standards of ecological accounting. Eco-Management and Auditing, 4(3), 87-97.

Schultze, W., \& Trommer, R. (2012). The concept of environmental performance and its measurement in empirical studies. Journal of Management Control, 22 (4), 375-412.

Spence, M. (1973). Job market signaling. The Quarterly Journal of Economics, 87 (3), 355-374.

Stanworth, J., \& Dandridge, T. (1994). Business franchising and economic change: an overview. International Small Business Journal, 12(2), 12-14.

Tassigny, M., Silva, I., Brasil, M., Bizarria, F., \& Frota, A. (2015). O mercado de franquias e sua contribuição econômica e socioambiental. Electronic Journal of Management, Education and Environmental Technology, 19(2), 703-716.

Testa, M., \& D’Amato, A. (2017). Corporate environmental responsibility and financial performance: does bidirectional causality work? Empirical evidence from the manufacturing industry. Social Responsibility Journal, 13(2), 221-234.

Thakore, R., Lowe, C., \& Nicholls, T. (2012) Financial impact of certified ISO 14001 Environment Management Systems in UK and Ireland, ICSDEC 2012. Anais. Recuperado em: 10 jan.2015, de: http://ascelibrary.org/doi/abs/10.1061/9780784412688.107. 
Toledo, G., \& Proença, C. (2005). Fatores críticos de sucesso da franquia - uma análise sob a óptica de ex-franqueados no município de São Paulo. Revista de Gestão, 12 (1), 43-53.

Van Kooten, G., Nelson, H., \& Vertinsky, I. (2005). Certification of sustainable forest management practices: a global perspective on why countries certify. Forest Policy and Economics, 7, 857-67.

Vance, P., Fávero, L., \& Luppe, M. (2008). Franquia empresarial: um estudo das características do relacionamento entre franqueadores e franqueados no Brasil. Revista de Administração, 43(1), 5971. Recuperado em: 10 jan.2015, de: http://www.revistasusp.sibi.usp.br/scielo.php?pid=S0080$21072008000100005 \&$ script $=$ sci_arttext

Varian, H. (1996). Intermediate Microeconomics. (4 ed.) Nova Iorque: W. W. Norton \& Company.

Wiengarten, F., Lo, C. K., \& Lam, J. Y. (2017). How does sustainability leadership affect firm performance? The choices associated with appointing a chief officer of corporate social responsibility. Journal of Business Ethics, 140(3), 477-493.

\section{NOTAS}

${ }^{1}$ Endrikat et al. (2014) complementam esta visão propondo um elo de retroalimentação positiva entre desempenho ambiental e resultados corporativos. Desta forma, teríamos um mecanismo de incentivos autorreforçador.

${ }^{2}$ Esta é uma visão empírica pautada por análises dos efeitos de certificações ISO 9000. Não obstante, espera-se que um efeito relevante destas defasagens possa ser observado para o caso de sinais econômicos atrelados aos mecanismos de sinalização de ações voltadas à gestão ambiental.

${ }^{3}$ Foram realizadas tentativas de obtenção de dados por meio de fontes externas, como dados da Exame Maiores e Melhores, Global Reporting Initiative e Base de Certificações do Inmetro. Não obstante, tais fontes não demonstraram uma cobertura adequada da amostra de franquias incluída neste estudo.

${ }^{4}$ Desta forma, permite-se excluir problemas associados a dupla contagem de ações de gestão ambiental.

Data da submissão: 17/03/2017

Data de aceite: $13 / 09 / 2017$ 